\title{
THE EFFECTS OF MITRAL VALVULOPLASTY ON CARDIOVASCULAR AND RENAL FUNCTION AT REST AND DURING EXERCISE ${ }^{1}$

\author{
By WALTER E. JUDSON, J. D. HATCHER, ${ }^{2}$ WILLIAM HOLLANDER, ${ }^{3}$ AND \\ MEYER H. HALPERIN
}

\author{
(From the Evans Memorial, Massachusetts Memorial Hospitals, and the Department of Medi- \\ cine, Boston University School of Medicine, Boston, Mass.)
}

(Submitted for publication December 27, 1954 ; accepted April 20, 1955)

The effects of mitral valvuloplasty on general cardiohemodynamic function in patients with mitral stenosis have been reported by many investigators (1-10). Measurements of renal blood flow, glomerular filtration rate, and excretion of sodium and their correlation with changes in cardiac output, blood oxygen measurements, and vascular pressures also have been extensively studied in patients with various types of heart disease, with and without congestive failure, at rest $(11,12)$ and during exercise (13-15). However, little information is available on the changes in renal circulation or excretion of electrolytes and water in the period early after mitral valvuloplasty (16). The purpose of this paper is to describe these changes in renal function and to correlate them with alterations in cardiovascular responses. Particular effort has been made to determine whether a specific cardiohemodynamic pattern is associated with disturbances of salt and water excretion before and after mitral valvuloplasty, both at rest and during exercise.

\section{METHODS AND MATERIALS}

Nine patients who had mitral stenosis and, in some instances, minimal aortic valvular disease, were studied before and after mitral valvuloplasty. A complete clinical description of the cardiovascular status and treatment of each of the patients is indicated in Table $I$. The laboratory procedures were performed before, and from 2 to 32 weeks after the operation.

The patients were studied in the postabsorptive state without any sedative medication. A double-lumen intracardiac catheter was placed in the right heart and pulmonary artery. An inlying needle was maintained in the

1 This investigation was supported in part by a grant from the National Heart Institute of the National Institutes of Health, U.S.P.H.S.

2 Present address : Queen's University, Kingston, Ontario, Canada.

${ }^{8}$ United States Public Health Service Fellow in Medicine, Evans Memorial, Massachusetts Memorial Hospitals. brachial artery. Zero point of reference of the pressures was $10 \mathrm{~cm}$. anterior to the back of the patient in the supine position. Pressures were measured by electromanometers 4 and recorded by a direct-writing oscillograph. Mean pressures were determined by electrical integration.

The resistances were calculated from the following formulae: ${ }^{5}$

The "total pulmonary" resistance (actually, total resistance opposing the right ventricle) was obtained from the following equation:

$$
R=\frac{P A_{m}}{C O} \times 1,332 \text { dynes seconds } \mathrm{cm} .^{-5} .
$$

The "pulmonary arteriolar" resistance (actually, the total pulmonary vascular resistance) was calculated as follows:

$$
\mathrm{R}=\frac{\mathrm{PA}_{\mathrm{m}}-{ }^{\prime} \mathrm{PC} \mathrm{C}_{\mathrm{m}} "}{\mathrm{CO}} \times 1,332 \text { dynes seconds } \mathrm{cm} .{ }^{-5} \text {. }
$$

The "total peripheral" resistance (actually, total resistance opposing the left ventricle) was calculated as follows:

$$
R=\frac{B A_{m} \times 1,332}{C O} \text { dynes seconds } \mathrm{cm} .^{-5} \text {. }
$$

4 Sanborn Company, Cambridge, Massachusetts.

5 Vascular resistances were calculated according to formulae devised by Gorlin and his co-workers (17), whose designations are employed here. "Total pulmonary" resistance is defined in terms of the gradient of pressure from the pulmonary arteries to zero, and actually includes the resistance of the pulmonary vessels, that of the mitral valve, as well as the resistance to filling by the left ventricle. Thus, the so-called "total pulmonary" resistance should more correctly be designated as the total resistance opposing the right ventricle. Similarly, calculation of the "pulmonary arteriolar" resistance is based on the pressure gradient between the pulmonary arteries and the "wedge-pressure." If the latter reflects pulmonary venous pressure, then the calculation of "pulmonary arteriolar" resistance actually includes the resistance of the capillaries and venules, and should be called total pulmonary vascular resistance. "Total peripheral" resistance, furthermore, is calculated on the basis of the pressure drop from the large arteries to zero, and actually includes the resistance to filling by the right heart. Therefore, this should more correctly be defined as the total resistance opposing the left ventricle. 
The work of the right and left ventricles against pressure was calculated with the following formulae:

$$
\begin{aligned}
& \mathrm{W}_{\mathrm{R}}=\frac{(\mathrm{CI} \times 1.055)\left(\left[\mathrm{PA}_{\mathrm{m}}-\mathrm{RA}_{\mathrm{m}}\right] \times 13.6\right)}{1,000} \\
& \mathrm{~W}_{\mathrm{L}}=\frac{(\mathrm{CI} \times 1.055)\left(\left[\mathrm{BA}_{\mathrm{m}}-5\right] \times 13.6\right.}{1,000 \mathrm{k} . \text { per minute per } \mathrm{M}^{2},} \\
& \text { kg. M. per minute per } \mathrm{M}^{2},
\end{aligned}
$$

$\mathrm{PA}_{\mathrm{m}}=$ pulmonary arterial mean pressure in $\mathrm{mm} . \mathrm{Hg}$, " $\mathrm{PC}_{\mathrm{m}}$ " = "pulmonary capillary" mean pressure in $\mathrm{mm} . \mathrm{Hg}$,

$\mathrm{BA}_{\mathrm{m}}=$ brachial arterial mean pressure in $\mathrm{mm} . \mathrm{Hg}$,

$\mathrm{CO}=$ cardiac output in $\mathrm{ml}$. per second,

$1,332=$ factor for converting $\mathrm{mm} . \mathrm{Hg}$ to dynes per $\mathrm{cm} .{ }^{2}$,

$\mathrm{W}_{\mathbf{R}}=$ work of right ventricle against pressure,

$\mathrm{W}_{\mathrm{L}}=$ work of left ventricle against pressure,

$\mathrm{CI}=$ cardiac index, liters per minute per $\mathbf{M}^{2}$,

$\mathrm{RA}_{\mathrm{m}}=$ right atrial pressure in $\mathrm{mm} . \mathrm{Hg}$,

$1,055=$ specific gravity of blood,

$5=$ assumed left ventricular diastolic pressure in $\mathrm{mm} . \mathrm{Hg}$,

13.6 = specific gravity of mercury.

An indwelling catheter was placed in the urinary bladder. After standard priming doses, a sustaining infusion containing inulin ( 3.3 per cent) and para-aminohippurate ( 0.5 per cent) in 3.5 per cent glucose and water was started and given at the rate of $1.2 \mathrm{ml}$. per minute by means of a calibrated drip.

After a preliminary practice run, at least one and usually two resting cardiac output determinations were made by the direct Fick method. Expired air was collected for two minutes in a Douglas bag while simultaneous blood samples were obtained at a constant rate from the brachial and pulmonary arteries. Immediately before and after the Fick procedure, pressures in the pulmonary artery, right ventricle, and brachial artery were recorded. The resting "pulmonary capillary" pressure was measured after the completion of measurement of the resting cardiac outputs. The volume of inspired air was measured by a Tissot spirometer which supplied outdoor air to the patient. Oxygen and carbon dioxide concentrations in the expired air were determined by the Haldane method (18), duplicate determinations being required to agree within 0.03 vol. per cent. Blood samples were analyzed for oxygen content, capacity and saturation by the method of Van Slyke and Neill (19). Blood oxygen contents were corrected for differences in hematocrit, duplicate determinations being required to agree within 0.10 vol. per cent.

During the control period, three to four urine collections were obtained. Urine was collected at the end of each 10 to 15 -minute period by washing out the bladder with $20 \mathrm{ml}$. of distilled water and evacuating the residual urine by displacement with air. Every twenty minutes arterial blood samples were obtained for the determinations of inulin, para-amino hippuric acid, chloride, sodium, potassium, and hematocrit. Blood and urine concentrations of para-amino hippuric acid were measured by the method of Goldring and Chasis (20) and inulin was determined by the method of Schreiner (21) or Roe, Ep- stein, and Goldstein (22). Serum and urinary sodium and potassium were determined by the internal standard flame photometer (lithium). Serum and urinary chloride were analyzed by the method of Wilson and Ball (23). Hematocrit was measured in Wintrobe tubes.

Measurements during the effects of exercise were carried out as follows :

With the distal lumen of the catheter wedged in a radicle of the pulmonary artery the patient exercised in the supine position by alternate straight leg-raising from two and one-half to nine and one-half minutes. During the final minute of exercise the cardiac output was measured. At the instant that the exercise was stopped the measurements of brachial artery, "pulmonary capillary," pulmonary arterial, and right ventricular pressures which were being taken were recorded for use in the calculation of the hemodynamic data. (Pressure measurements were also taken and recorded repeatedly during exercise but were not regarded as completely valid because of the marked fluctuations in intrathoracic and intraabdominal pressures associated with the strenuous exercise of alternate straight leg-raising.) Urine collections were made at the end of the 10-minute period including the time during which the exercise was performed ("the exercise period") and thereafter for three 10 to 15 -minute periods. All these procedures were performed after operation in the identical manner as before.

\section{RESULTS}

Cardiohemodynamic data for each patient are presented in Table II, and the results in two cases are shown graphically in Figures 1 and 2 . The mean data for the group and a statistical analysis are shown in Table III, which is the major basis for the following presentation.

\section{Cardiovascular responses}

Cardiac index and oxygen measurements. The mean resting cardiac index for the entire group before the operation was 2.71 liters per minute per square meter of body surface. The mean increase in the resting cardiac index for the group after operation was 0.36 liter per minute per square meter of body surface, which was not statistically significant. The mean cardiac index during exercise was $0.76 \mathrm{~L}$. per min. per $\mathrm{M}^{2}$ greater after than before operation. In response to exercise all but one patient (C. J.) had rises in cardiac index both before and after operation (average $0.87 \mathrm{~L}$. per min. per $\mathrm{M}^{2}$ preoperatively and $1.26 \mathrm{~L}$. per min. per $\mathrm{M}^{2}$ postoperatively). Thus, the response of cardiac index to exercise after operation averaged $0.41 \mathrm{~L}$. per min. per $\mathrm{M}^{2}$ greater than 


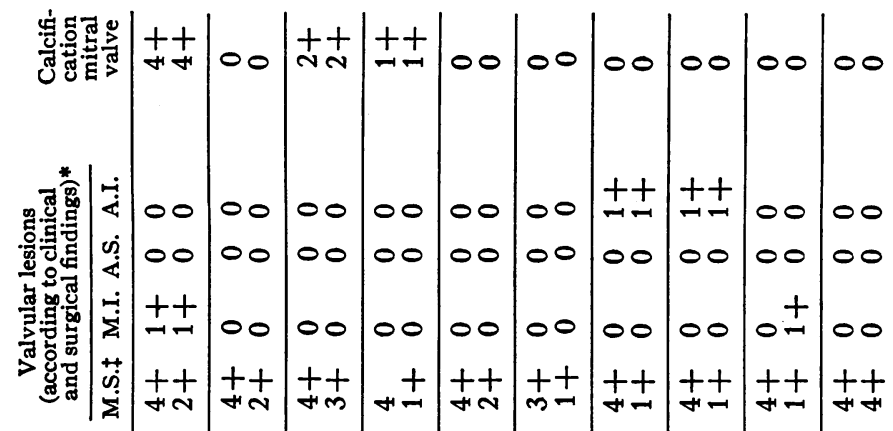


TABLE II

Individual cardiohemodynamic data *

\begin{tabular}{|c|c|c|c|c|c|c|c|c|c|c|c|c|c|}
\hline \multirow{2}{*}{$\begin{array}{c}\text { Patient } \\
\text { Age Sex } \\
\text { Body surface } \\
\text { area } \\
\left(M^{2}\right)\end{array}$} & \multirow{2}{*}{$\begin{array}{l}\text { Time } \\
\text { relative to } \\
\text { operation }\end{array}$} & \multicolumn{2}{|c|}{$\begin{array}{c}\text { Arterial } \\
\text { oxygen } \\
\text { saturation } \\
\%\end{array}$} & \multicolumn{2}{|c|}{$\begin{array}{c}\text { Oxygen } \\
\text { consumption } \\
m l . / m i n . / M^{2}\end{array}$} & \multicolumn{2}{|c|}{$\begin{array}{c}\text { A-V oxygen } \\
\text { difference } \\
\text { vol. \% }\end{array}$} & \multicolumn{2}{|c|}{$\begin{array}{l}\text { Cardiac index } \\
L . / \min . / M^{2}\end{array}$} & \multicolumn{2}{|c|}{$\begin{array}{l}\text { Heart rate } \\
\text { beats/min. }\end{array}$} & \multicolumn{2}{|c|}{$\begin{array}{l}\text { Stroke index } \\
\mathrm{ml} . / \mathrm{beat} / \mathrm{M}^{2}\end{array}$} \\
\hline & & $\mathbf{R}$ & $\mathrm{E}$ & $\mathbf{R}$ & $\mathbf{E}$ & $\mathbf{R}$ & $\mathbf{E}$ & $\mathbf{R}$ & $\mathbf{E}$ & $\mathbf{R}$ & $\mathbf{E}$ & $\mathbf{R}$ & $\mathbf{E}$ \\
\hline $43{ }_{1.99}^{\mathrm{J} \cdot \mathrm{C} .} \mathrm{M}$ & Pre-op. & 91 & 90 & 163 & 347 & 5.23 & 10.69 & 3.18 & 3.23 & 85 & 100 & 37 & 32 \\
\hline 1.96 & $\begin{array}{l}16 \text { wks. } \\
\text { Post-op. }\end{array}$ & 94 & 95 & 164 & 350 & 5.07 & 8.48 & 3.26 & 4.12 & 66 & 98 & 49 & 42 \\
\hline $36{ }_{1.64}^{\text {A. L. }}$ & Pre-op. & 99 & 100 & 148 & 427 & 5.86 & 10.67 & 2.54 & 4.00 & 84 & 180 & 30 & 22 \\
\hline 1.63 & $\begin{array}{l}9 \text { wks. } \\
\text { Post-op. }\end{array}$ & 97 & 99 & 144 & 427 & 5.03 & 10.48 & 2.85 & 4.07 & 92 & 140 & 31 & 29 \\
\hline $40{ }_{1.63}^{\text {C. J. }} \mathrm{F}$ & Pre-op. & 89 & 86 & 180 & 209 & 9.04 & 10.92 & 1.98 & 1.91 & 107 & 124 & 18 & 15 \\
\hline 1.57 & $\begin{array}{l}2 \text { wks. } \\
\text { Post-op. }\end{array}$ & 92 & 95 & 182 & 321 & 6.24 & 11.46 & 2.93 & 2.80 & 84 & 98 & 35 & 29 \\
\hline 37 C. S. $\mathrm{M}$ & Pre-op. & 93 & 90 & 179 & 427 & 6.73 & 13.36 & 2.67 & 3.19 & 85 & 136 & 31 & 23 \\
\hline 1.71 & $\begin{array}{l}28 \text { wks. } \\
\text { Post-op. }\end{array}$ & 98 & 98 & 171 & 439 & 4.13 & 7.45 & 4.17 & 5.89 & 80 & 110 & 52 & 54 \\
\hline $48_{1.66}^{\text {E. M. }}$ & Pre-op. & 89 & 86 & 127 & - & 5.66 & 9.71 & 2.24 & 一 & 77 & 104 & 29 & - \\
\hline 1.63 & $\begin{array}{l}20 \text { wks. } \\
\text { Post-op. }\end{array}$ & 95 & 93 & 131 & 363 & 4.80 & 9.35 & 2.74 & 3.88 & 71 & 120 & 39 & 32 \\
\hline $49_{1.69}^{\text {C. M. }}$ & Pre-op. & 100 & 92 & 130 & 367 & 4.21 & 7.51 & 3.09 & 4.89 & 63 & 98 & 49 & 50 \\
\hline 1.69 & $\begin{array}{l}32 \text { wks. } \\
\text { Post-op. }\end{array}$ & 99 & 99 & 125 & 379 & 5.04 & 9.06 & 2.48 & 4.19 & 60 & 84 & 41 & 50 \\
\hline $22_{1.49}^{\text {E. T. }}$ & Pre-op. & 94 & 92 & 146 & 377 & 4.42 & 8.24 & 3.28 & 4.58 & 62 & 110 & 53 & 42 \\
\hline 1.48 & $\begin{array}{l}17 \text { wks. } \\
\text { Post-op. }\end{array}$ & 99 & - & 144 & 357 & 3.75 & 6.65 & 3.86 & 5.36 & 78 & 96 & 50 & 56 \\
\hline${ }_{50}^{\text {E. C. }}$ & Pre-op. & 95 & 100 & 130 & 280 & 6.22 & 11.13 & 2.14 & 2.51 & 48 & 88 & 44 & 28 \\
\hline 1.44 & $\begin{array}{l}2 \text { wks. } \\
\text { Post-op. }\end{array}$ & 96 & 94 & 149 & 344 & 5.12 & 9.41 & 2.87 & 3.65 & 82 & 100 & 35 & 37 \\
\hline $40_{2.10}^{\text {A. C. }} \mathrm{M}$ & Pre-op. & 96 & 94 & 145 & 470 & 4.45 & 9.83 & 3.25 & 4.79 & 78 & 138 & 42 & 35 \\
\hline 2.08 & $\begin{array}{l}6 \text { wks. } \\
\text { Post-op. }\end{array}$ & 94 & 94 & 124 & 538 & 4.97 & 10.65 & 2.48 & 5.06 & 68 & 120 & 36 & 42 \\
\hline${ }_{1.46}^{\text {A. S. } †}$ F & Pre-op. & 97 & 99 & 160 & 286 & 4.59 & 7.84 & 3.49 & 3.65 & 84 & 112 & 42 & 33 \\
\hline 1.46 & $\begin{array}{c}16 \text { wks. } \\
\text { Post-op. } \\
\text { (Cardiotomy) }\end{array}$ & 96 & 98 & 172 & 425 & 4.67 & 7.99 & 3.67 & 5.29 & 79 & 128 & 46 & 41 \\
\hline $\begin{array}{rl}* & R= \\
E & = \\
S & = \\
D & = \\
M & =\end{array}$ & $\begin{array}{l}\text { Rest } \\
\text { Exercise } \\
\text { Systolic } \\
\text { Diastolic } \\
\text { Mean }\end{array}$ & & & & & $\begin{array}{l}\dagger \text { Not } \\
\mathrm{M}^{2}-\mathrm{re}\end{array}$ & $\begin{array}{l}\text { includec } \\
\text { efers to }\end{array}$ & $\begin{array}{l}n \text { mean } \\
\text { uare me }\end{array}$ & $\begin{array}{l}\text { or stat } \\
\text { eters of }\end{array}$ & $\begin{array}{l}\text { cal sur } \\
\text { dy sur }\end{array}$ & $\begin{array}{l}\text { mmary } \\
\text { face a }\end{array}$ & & \\
\hline
\end{tabular}


TABLE II-Continued

\begin{tabular}{|c|c|c|c|c|c|c|c|c|c|c|c|c|c|c|c|c|c|}
\hline \multirow{4}{*}{$\begin{array}{c}\text { Patient } \\
\text { Age Sex } \\
\text { Body surface } \\
\text { area } \\
\left(M^{2}\right)\end{array}$} & \multirow{4}{*}{$\begin{array}{l}\text { Time } \\
\text { relative to } \\
\text { operation }\end{array}$} & \multicolumn{16}{|c|}{ Pressures $(m m . H g)$} \\
\hline & & \multicolumn{6}{|c|}{ Pulmonary artery } & \multirow{2}{*}{\multicolumn{2}{|c|}{$\begin{array}{c}\text { Mean } \\
\text { "pulmonary } \\
\text { capillary" }\end{array}$}} & \multirow{2}{*}{\multicolumn{2}{|c|}{$\begin{array}{l}\text { Right ven- } \\
\text { tricular end } \\
\text { diastolic }\end{array}$}} & \multicolumn{6}{|c|}{ Systemic arterial } \\
\hline & & \multicolumn{3}{|c|}{$\mathbf{R}$} & \multicolumn{3}{|c|}{$\mathrm{E}$} & & & & & \multicolumn{3}{|c|}{$\mathbf{R}$} & \multicolumn{3}{|c|}{$E$} \\
\hline & & $\mathrm{S}$ & $\mathbf{D}$ & $\mathbf{M}$ & $\mathrm{s}$ & $\mathrm{D}$ & $\mathbf{M}$ & $\mathbf{R}$ & $\mathrm{E}$ & $\mathbf{R}$ & $\mathrm{E}$ & $\mathrm{s}$ & D & $\mathbf{M}$ & $\mathbf{S}$ & $\mathbf{D}$ & $\mathbf{M}$ \\
\hline $\begin{array}{c}\mathrm{J} \cdot \mathrm{C} . \\
1.99\end{array}$ & Pre-op. & 90 & 45 & 55 & 115 & 55 & 85 & 22 & 32 & 10 & 15 & 120 & 70 & 85 & 145 & 80 & 95 \\
\hline 1.96 & $\begin{array}{l}16 \text { wks. } \\
\text { Post-op. }\end{array}$ & 58 & 28 & 40 & 80 & 40 & 53 & 20 & 30 & 12 & 15 & 130 & 75 & 90 & 160 & 100 & 120 \\
\hline $36{ }_{1.64}^{\text {A. L. }} \mathrm{F}$ & Pre-op. & 35 & 20 & 24 & 63 & 40 & 43 & 21 & 30 & 15 & 30 & 140 & 90 & 110 & 178 & 110 & 130 \\
\hline 1.63 & $\begin{array}{l}9 \text { wks: } \\
\text { Post-op. }\end{array}$ & 33 & 15 & 22 & 48 & 30 & 37 & 14 & 27 & 10 & 12 & 130 & 75 & 90 & 187 & 115 & 140 \\
\hline $40_{1.63}^{\text {C. J. }}$ & Pre-op. & 125 & 70 & 90 & 175 & 80 & 115 & 25 & 35 & 25 & - & 190 & 120 & 150 & 200 & 135 & 160 \\
\hline 1.57 & $\begin{array}{l}2 \text { wks. } \\
\text { Post-op. }\end{array}$ & 78 & 40 & 52 & 110 & 52 & 72 & 19 & 35 & 12 & 26 & 120 & 80 & 100 & 170 & 115 & 125 \\
\hline $37_{1.64} \begin{array}{c}\text { C. S. } \\
M\end{array}$ & Pre-op. & 102 & 47 & 65 & 138 & 61 & 99 & 30 & 41 & 10 & 18 & 130 & 80 & 100 & 170 & 100 & 135 \\
\hline 1.71 & $\begin{array}{l}28 \text { wks. } \\
\text { Post-op. }\end{array}$ & 50 & 28 & 38 & 80 & 48 & 60 & 22 & 30 & 5 & 10 & 120 & 60 & 90 & 145 & 70 & 100 \\
\hline $48_{1.66}^{\text {E. M. }}$ & Pre-op. & 76 & 38 & 55 & 110 & 52 & 75 & 34 & 55 & 5 & 10 & 170 & 90 & 120 & 265 & 150 & 207 \\
\hline 1.63 & $\begin{array}{l}20 \text { wks. } \\
\text { Post-op. }\end{array}$ & 47 & 28 & 35 & 88 & 48 & 57 & 28 & 42 & 5 & 12 & 190 & 100 & 130 & 210 & 110 & 145 \\
\hline${ }_{49}^{\text {C. M. }}{ }_{1.69}^{M}$ & Pre-op. & 65 & 30 & 40 & 112 & 52 & 85 & 26 & 55 & 5 & 10 & 135 & 75 & 100 & 180 & 90 & 130 \\
\hline 1.69 & $\begin{array}{l}32 \text { wks. } \\
\text { Post-op. }\end{array}$ & 32 & 16 & 23 & 60 & 35 & 45 & 15 & 22 & 5 & 5 & 125 & 65 & 90 & 145 & 75 & 110 \\
\hline${ }_{22}^{\text {E. T. }}{ }_{1.49} \mathrm{~F}$ & Pre-op. & 45 & 20 & 33 & 82 & 46 & 65 & 20 & 40 & 2 & 5 & 105 & 60 & 80 & 125 & 72 & 88 \\
\hline 1.48 & $\begin{array}{l}17 \text { wks. } \\
\text { Post-op. }\end{array}$ & 42 & 22 & 32 & 58 & 30 & 43 & 18 & 36 & 5 & 5 & 122 & 62 & 79 & - & - & - \\
\hline${ }_{50}^{\text {E. C. }}$ & Pre-op. & 50 & 30 & 40 & 90 & 55 & 68 & 20 & 38 & 10 & 15 & 105 & 60 & 75 & - & - & 一 \\
\hline 1.44 & $\begin{array}{l}2 \text { wks. } \\
\text { Post-op. }\end{array}$ & 53 & 28 & 35 & 86 & 40 & 56 & 18 & 21 & 8 & 10 & 100 & 60 & 75 & 105 & 65 & 80 \\
\hline $40_{2.10}^{\text {A. C. }}$ & Pre-op. & 45 & 35 & 40 & 90 & 60 & 68 & 30 & 48 & 5 & 20 & 138 & 80 & 100 & 140 & 80 & 100 \\
\hline 2.08 & $\begin{array}{l}6 \text { wks. } \\
\text { Post-op. }\end{array}$ & 33 & 20 & 25 & 58 & 38 & 47 & 15 & 21 & 5 & 10 & 140 & 90 & 110 & 160 & 90 & 120 \\
\hline${ }_{39_{1.46}^{\text {A. S. }}}$ & Pre-op. & 50 & 28 & 35 & 70 & 34 & 50 & 18 & 28 & 5 & 10 & 120 & 70 & 95 & 155 & 90 & 120 \\
\hline 1.46 & $\begin{array}{c}16 \text { wks. } \\
\text { Post-op. } \\
\text { (Cardiotomy) }\end{array}$ & 45 & 23 & 33 & 75 & 38 & 55 & 20 & 30 & 4 & 9 & 130 & 80 & 100 & - & - & - \\
\hline
\end{tabular}




\begin{tabular}{|c|c|c|c|c|c|c|c|c|c|c|c|}
\hline \multirow{3}{*}{$\begin{array}{c}\text { Patient } \\
\text { Age Sex } \\
\text { Body surface } \\
\text { area } \\
\left(M^{2}\right)\end{array}$} & \multirow{3}{*}{$\begin{array}{c}\text { Time } \\
\text { relative to } \\
\text { operation }\end{array}$} & \multicolumn{6}{|c|}{$\begin{array}{l}\text { Resistances } \\
\text { dynes seconds } \mathrm{cm}^{-6}\end{array}$} & \multicolumn{4}{|c|}{$\begin{array}{l}\text { Work of ventricles } \\
\text { against pressure } \\
\mathrm{kg} . \mathrm{M}_{\mathrm{g}} / \mathrm{min} . / \mathrm{M}^{2}\end{array}$} \\
\hline & & \multicolumn{2}{|c|}{$\begin{array}{l}\text { "Total } \\
\text { pulmonary" }\end{array}$} & \multicolumn{2}{|c|}{$\begin{array}{l}\text { "Pulmonary } \\
\text { arteriolar" }\end{array}$} & \multicolumn{2}{|c|}{$\begin{array}{c}\text { Total } \\
\text { peripheral }\end{array}$} & \multicolumn{2}{|c|}{$\begin{array}{c}\text { Right } \\
\text { ventricle }\end{array}$} & \multicolumn{2}{|c|}{$\begin{array}{c}\text { Left } \\
\text { ventricle }\end{array}$} \\
\hline & & $\mathbf{R}$ & $E$ & $\mathbf{R}$ & E & $\mathbf{R}$ & $\mathbf{E}$ & $\mathbf{R}$ & $\bar{E}$ & $\mathbf{R}$ & $\bar{E}$ \\
\hline $43{ }_{1.99}^{\mathrm{J} \cdot \mathrm{C} .} \mathrm{M}$ & Pre-op. & 700 & 1,050 & 420 & 660 & 1,080 & 1,180 & 2.0 & 3.3 & 3.6 & 4.2 \\
\hline 1.96 & $\begin{array}{l}16 \text { wks. } \\
\text { Post-op. }\end{array}$ & 500 & 520 & 250 & 230 & 1,130 & 1,190 & 1.3 & 2.2 & 4.0 & 6.8 \\
\hline $36{ }_{1.64}^{\text {A. L. }} \mathrm{F}$ & Pre-op. & 460 & 520 & 60 & 160 & 2,110 & 1,580 & 0.3 & 0.8 & 3.8 & 7.2 \\
\hline 1.63 & $\begin{array}{l}9 \text { wks. } \\
\text { Post-op. }\end{array}$ & 380 & 450 & 140 & 120 & 1,550 & 1,690 & 0.5 & 1.5 & 3.5 & 7.9 \\
\hline $40{ }_{1.63}^{C . J .} F$ & Pre-op. & 2,230 & 2,950 & 1,610 & 2,050 & 3,710 & 4,100 & 1.8 & - & 4.1 & 4.2 \\
\hline 1.57 & $\begin{array}{l}2 \text { wks. } \\
\text { Post-op. }\end{array}$ & 900 & 1,310 & 570 & 670 & 1,740 & 2,270 & 1.7 & 1.8 & 4.0 & 4.8 \\
\hline $37{ }_{1.64}^{\text {C. S. }} \mathrm{M}$ & Pre-op. & 1,190 & 1,510 & 640 & 890 & 1,830 & 2,060 & 2.1 & 3.7 & 3.6 & 6.0 \\
\hline 1.71 & $\begin{array}{l}28 \text { wks. } \\
\text { Post-op. }\end{array}$ & 430 & 480 & 180 & 240 & 1,010 & 790 & 2.0 & 4.2 & 5.1 & 8.0 \\
\hline $48_{1.66}^{\text {E. M. }}$ & Pre-op. & 1,180 & - & 450 & - & 2,580 & - & 1.6 & - & 3.7 & - \\
\hline 1.63 & $\begin{array}{l}20 \text { wks. } \\
\text { Post-op. }\end{array}$ & 630 & 720 & 130 & 190 & 2,320 & 1,830 & 1.2 & 2.5 & 4.9 & 7.8 \\
\hline${ }_{49}^{\text {C. M. }}{ }_{1.69}^{M}$ & Pre-op. & 610 & 820 & 210 & 290 & 1,530 & 1,260 & 1.6 & 5.3 & 4.2 & 8.8 \\
\hline 1.69 & $\begin{array}{l}32 \text { wks. } \\
\text { Post-op. }\end{array}$ & 440 & 510 & 150 & 260 & 1,720 & 1,240 & 0.6 & 2.4 & 3.0 & 6.3 \\
\hline${ }_{22}^{\text {E. T. }}{ }_{1.49}^{F}$ & Pre-op. & 540 & 760 & 210 & 290 & 1,310 & 1,030 & 1.4 & 3.9 & 3.5 & 5.4 \\
\hline 1.48 & $\begin{array}{l}17 \text { wks. } \\
\text { Post-op. }\end{array}$ & 450 & 430 & 200 & 70 & 1,110 & - & 1.5 & 2.9 & 4.4 & - \\
\hline${ }_{50}^{\text {E. C. }}{ }_{1.43} \mathrm{~F}$ & Pre-op. & 1,050 & 1,510 & 520 & 670 & 1,960 & - & 0.9 & 1.9 & 2.3 & - \\
\hline 1.44 & $\begin{array}{l}\text { 2 wks. } \\
\text { Post-op. }\end{array}$ & 680 & 850 & 330 & 530 & 1,460 & 1,220 & 1.1 & 2.4 & 2.9 & 4.2 \\
\hline $40_{2.10}^{\mathrm{A} . \mathrm{C} .} \mathrm{M}$ & Pre-op. & 470 & 540 & 120 & 160 & 1,170 & 800 & 1.6 & 3.3 & 4.4 & 6.5 \\
\hline 2.08 & $\begin{array}{l}\text { 6.wks. } \\
\text { Post-op. }\end{array}$ & 390 & 360 & 160 & 200 & 1,710 & 910 & 0.7 & 2.7 & 3.7 & 8.3 \\
\hline $39{ }_{1.46}^{\text {A. S. } †} F$ & Pre-op. & 550 & 750 & 270 & 330 & 1,490 & 1,800 & 1.5 & 2.1 & 4.5 & 6.0 \\
\hline 1.46 & $\begin{array}{c}16 \text { wks. } \\
\text { Post-op. } \\
\text { (Cardiotomy) }\end{array}$ & 490 & 570 & 190 & 260 & 1,490 & - & 1.5 & 3.5 & 5.0 & - \\
\hline
\end{tabular}




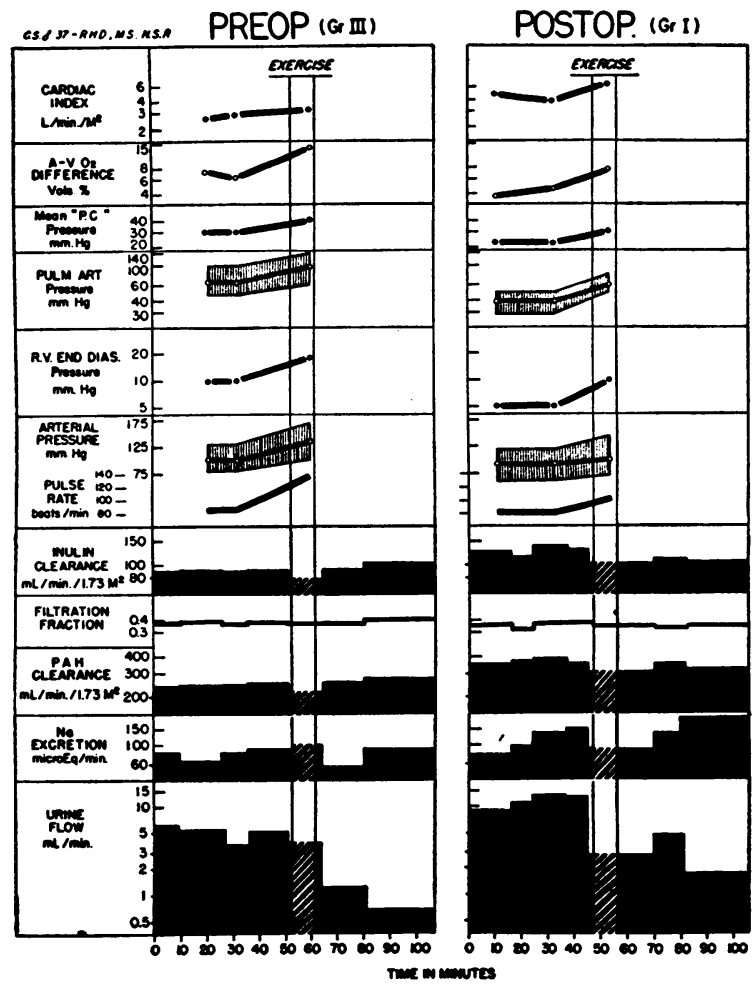

Fig. 1. Chart of the Cardiovascular and Renal Measurements at Rest and during Exercise in Patient C. S. with Mitral Stenosis before and after Mitral Valvuloplasty

Postoperatively this patient showed the greatest improvement in cardio-renal function both at rest and during exercise.

before operation, but this change lacked statistical significance.

The oxygen consumption of each patient at rest was almost identical before and after operation. With exercise the oxygen consumption tended to be higher after than before operation, perhaps reflecting ability to do a greater amount of work. This difference lacked statistical significance in the group as a whole. The arteriovenous oxygen differences both at rest and during exercise were on the average about 1 vol. per cent lower after operation than before. The range of the data was so wide, however, that for this small group statistical analysis did not indicate the change to be significant. The arterial oxygen saturation of seven of the nine patients decreased slightly with exercise preoperatively, but this occurred in only two patients postoperatively. Statistically these changes were not significant. However, in this part of the oxygen dis- sociation curve small differences in saturation may represent considerable differences in oxygen tension, which we did not measure directly.

\section{Vascular pressures}

Pulmonary arterial pressure. All of the patients had abnormally elevated pulmonary arterial pressures at rest both before (average $49 \mathrm{~mm}$. $\mathrm{Hg}$ ) and after operation (average $34 \mathrm{~mm} . \mathrm{Hg}$ ), this change being physiologically and statistically highly significant $(<\mathrm{P}=0.01)$. After exercise, the average mean pulmonary arterial pressure was $78 \mathrm{~mm}$. $\mathrm{Hg}$ before operation and $52 \mathrm{~mm}$. $\mathrm{Hg}$ after operation. All of the patients had a lower mean pulmonary arterial pressure in response to exercise after than before operation, the

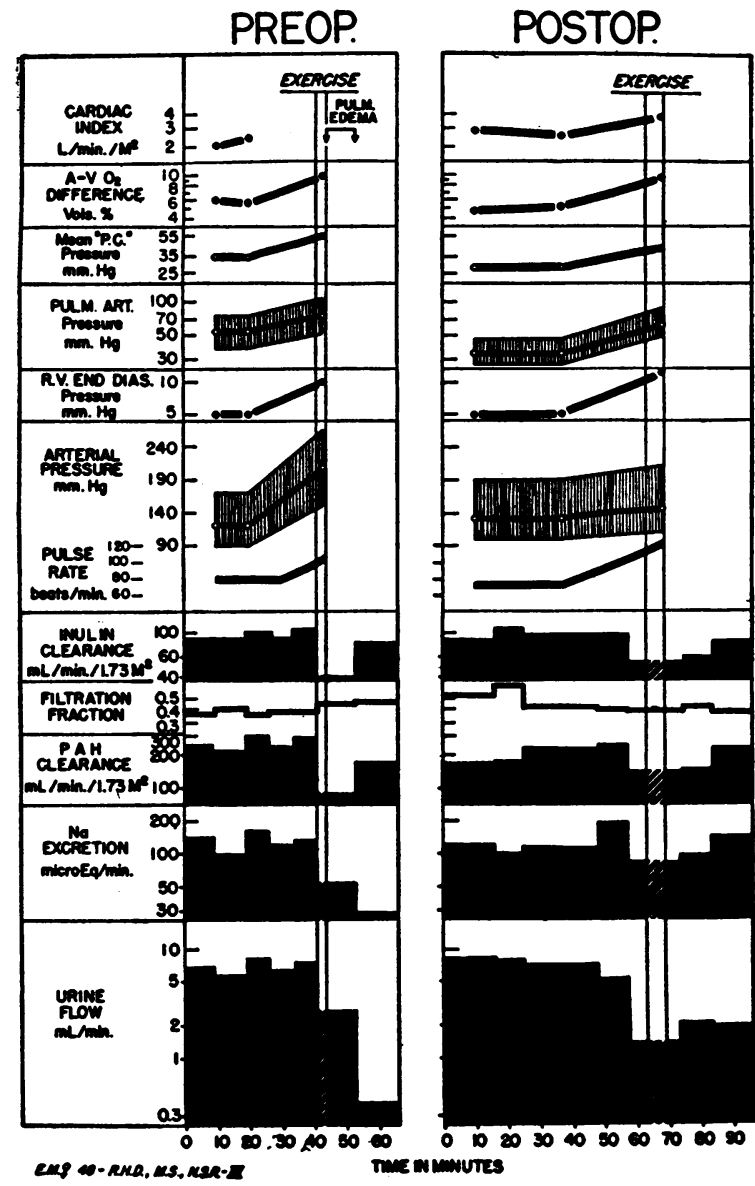

Fig. 2. Chart of the Cardiovascular and Renal Measurements at Rest and during Exercise in Patient E. M. with Mitral Stenosis before and after Mrtral Valvuloplasty

Preoperatively the patient developed pulmonary edema during exercise. 
decrease being 6 to $43 \mathrm{~mm}$. $\mathrm{Hg}$ (average $26 \mathrm{~mm}$. $\mathrm{Hg}$ ). In response to exercise, the mean pulmonary arterial pressure rise averaged $29 \mathrm{~mm}$. $\mathrm{Hg}$ (59 per cent) before operation and $19 \mathrm{~mm}$. $\mathrm{Hg}$ (56 per cent) after operation. All these changes had high physiologic and statistical significance.

"Pulmonary capillary" pressure. The average mean "pulmonary capillary" ("PC") pressure at rest before operation was $25 \mathrm{~mm}$. $\mathrm{Hg}$ and $19 \mathrm{~mm}$. $\mathrm{Hg}$ after operation. Postoperatively six of the nine patients showed a significant fall in mean pressures at rest $(6$ to $15 \mathrm{~mm}$. $\mathrm{Hg}$ ). In none of these patients, however, did the mean pressure return to normal. In response to exercise the mean "PC" pressure rose, on the average, to $42 \mathrm{~mm}$. $\mathrm{Hg}$ preoperatively and $29 \mathrm{~mm}$. $\mathrm{Hg}$ postoperatively, or an average of $13 \mathrm{~mm}$. $\mathrm{Hg}$ lower after operation, a change which was highly significant statistically. However, the average rise in mean "PC" pressure after exercise was $16 \mathrm{~mm}$.

TABLE III

Mean data and statistical analysis

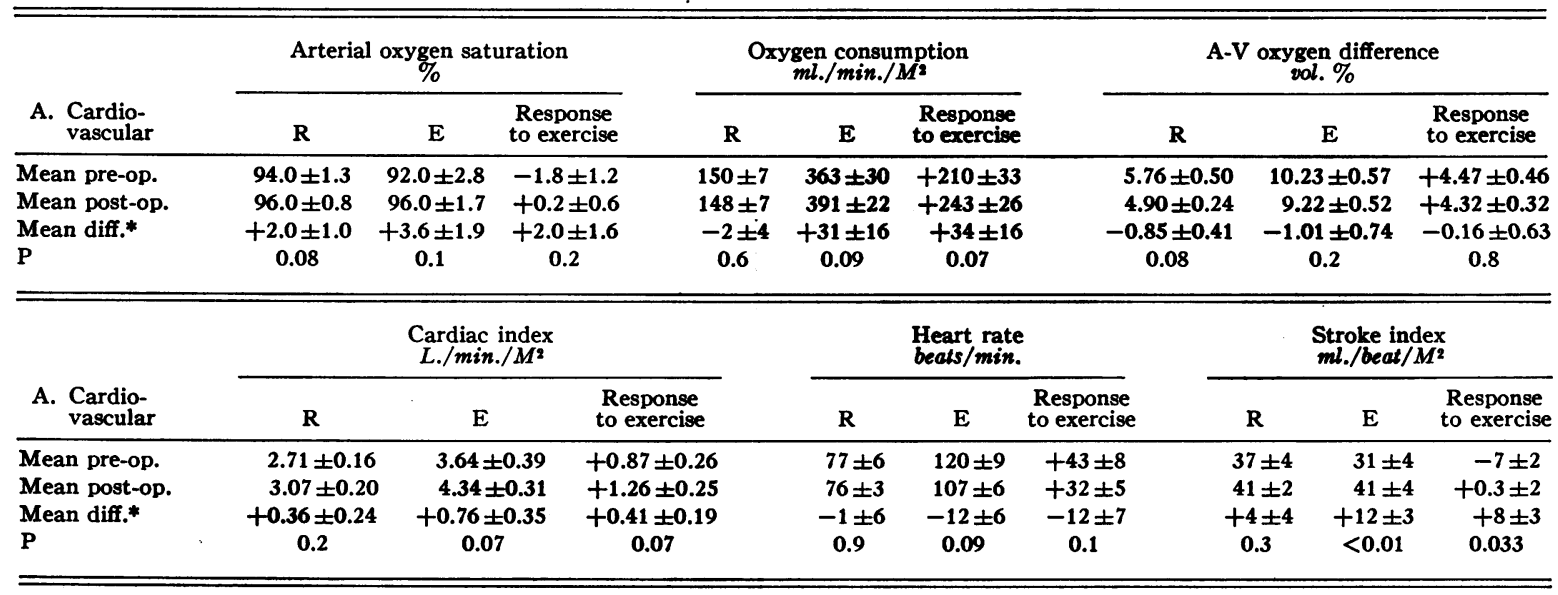

Pressures (mm. $\mathrm{Hg})$

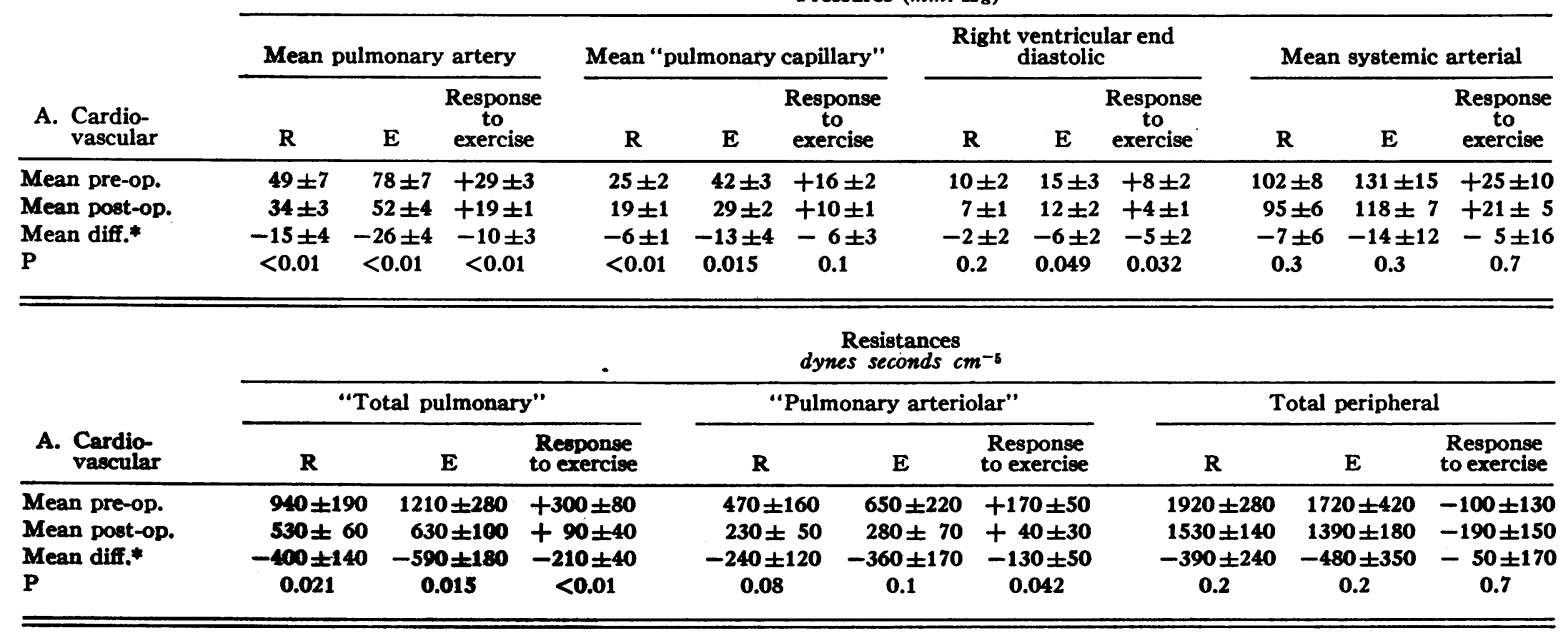

Work of ventricles against pressure 
TABLE III-Continued

\begin{tabular}{|c|c|c|c|c|c|c|c|c|c|c|c|c|}
\hline \multirow[b]{3}{*}{ B. Renal } & \multicolumn{12}{|c|}{$\begin{array}{c}\text { Clearance } \\
m \mathrm{~m} . / \mathrm{min} . / 1.73 \mathrm{M}^{2}\end{array}$} \\
\hline & \multicolumn{6}{|c|}{ PAH } & & \multicolumn{5}{|c|}{ Inulin } \\
\hline & \multicolumn{2}{|l|}{ Rest } & Exercise & $\begin{array}{l}\text { Response } \\
\text { to exercise }\end{array}$ & \multicolumn{2}{|c|}{ Recovery } & & Rest & Exercise & \multicolumn{2}{|c|}{$\begin{array}{l}\text { Response } \\
\text { to exercise }\end{array}$} & Recovery \\
\hline $\begin{array}{l}\text { Mean pre-op. } \\
\text { Mean post-op. } \\
\text { Mean diff. } \\
\text { P }\end{array}$ & \multicolumn{2}{|c|}{$\begin{array}{c}318 \pm 31 \\
370 \pm 47 \\
+42 \pm 38 \\
0.3\end{array}$} & $\begin{aligned} 195 \pm 31 \\
283 \pm 51 \\
+82 \pm 47 \\
0.1\end{aligned}$ & $\begin{aligned}-138 \pm 22 \\
-87 \pm 11 \\
-56 \pm 26 \\
0.08\end{aligned}$ & \multicolumn{2}{|c|}{$\begin{array}{c}294 \pm 35 \\
351 \pm 42 \\
+48 \pm 43 \\
0.3\end{array}$} & & $\begin{array}{c}100 \pm 7 \\
117 \pm 10 \\
+16 \pm 9 \\
0.1\end{array}$ & $\begin{aligned} & 64 \pm 10 \\
& 90 \pm 14 \\
&+24 \pm 22 \\
& 0.3\end{aligned}$ & \multicolumn{2}{|c|}{$\begin{array}{c}-38 \pm 12 \\
-26 \pm 6 \\
+12 \pm 16 \\
0.5\end{array}$} & $\begin{array}{c}102 \pm 8 \\
129 \pm 12 \\
+14 \pm 11 \\
0.3\end{array}$ \\
\hline & \multicolumn{4}{|c|}{$\begin{array}{l}\text { F.F. } \\
\%\end{array}$} & \multicolumn{4}{|c|}{$\begin{array}{c}\text { UV } \\
m l . / m i n .\end{array}$} & \multicolumn{4}{|c|}{$\begin{array}{c}\mathrm{UN}_{\mathrm{N}} \mathrm{V} \\
\text { MicroEq./min. }\end{array}$} \\
\hline B. Renal & Rest & $\begin{array}{l}\text { Exer- } \\
\text { cise }\end{array}$ & $\begin{array}{c}\text { Response } \\
\text { to } \\
\text { exercise }\end{array}$ & $\begin{array}{c}\mathrm{Re}- \\
\text { covery }\end{array}$ & Rest & $\begin{array}{l}\text { Exer- } \\
\text { cise }\end{array}$ & $\begin{array}{c}\text { Response } \\
\text { to } \\
\text { exercise }\end{array}$ & $\begin{array}{c}\text { Re- } \\
\text { covery }\end{array}$ & Rest & $\begin{array}{c}\text { Exer- } \\
\text { cise }\end{array}$ & $\begin{array}{c}\text { Response } \\
\text { to } \\
\text { exercise } \\
\end{array}$ & $\begin{array}{c}\text { Re- } \\
\text { covery }\end{array}$ \\
\hline $\begin{array}{l}\text { Mean pre-op. } \\
\text { Mean post-op. } \\
\text { Mean diff. } \\
\text { P }\end{array}$ & $\begin{array}{c}33 \pm 2 \\
34 \pm 1 \\
+1 \pm 2 \\
0.5\end{array}$ & $\begin{array}{r}34 \pm 5 \\
34 \pm 2 \\
0 \pm 3 \\
-\end{array}$ & $\begin{array}{r}+2 \pm 3 \\
+0.3 \pm 2 \\
-2 \pm 4 \\
0.6\end{array}$ & $\begin{array}{r}36 \pm 3 \\
34 \pm 3 \\
0 \pm 3 \\
-\end{array}$ & $\begin{array}{c}4.8 \pm 1.1 \\
6.7 \pm 1.4 \\
- \\
-\end{array}$ & $\begin{array}{c}1.7 \pm 0.5 \\
2.9 \pm 0.6 \\
- \\
-\end{array}$ & $\begin{array}{r}-4.0 \pm 0.9 \\
-4.0 \pm 1.3 \\
0.2 \pm 1.8 \\
>0.9\end{array}$ & $\begin{array}{c}2.5 \pm 1.3 \\
3.6 \pm 0.9 \\
- \\
-\end{array}$ & $\begin{array}{c}107 \pm 17 \\
125 \pm 23 \\
- \\
-\end{array}$ & $\begin{array}{c}62 \pm 11 \\
86 \pm 18 \\
- \\
-\end{array}$ & $\begin{array}{c}-45 \pm 17 \\
-40 \pm 11 \\
-4 \pm 14 \\
0.8\end{array}$ & $\begin{array}{c}101 \pm 39 \\
121 \pm 20 \\
- \\
-\end{array}$ \\
\hline \multirow[b]{2}{*}{ B. Renal } & \multicolumn{4}{|c|}{$\underset{\text { MicroEq./min. }}{\mathrm{U}_{\mathrm{KV}}}$} & \multicolumn{4}{|c|}{$\begin{array}{c}\mathrm{U}_{\mathrm{ClV}} \\
\text { MicroEq./min. }\end{array}$} & & & & \\
\hline & Rest & $\begin{array}{l}\text { Exer- } \\
\text { cise }\end{array}$ & $\begin{array}{c}\text { Response } \\
\text { to } \\
\text { exercise }\end{array}$ & $\begin{array}{c}\text { Re- } \\
\text { covery }\end{array}$ & Rest & $\begin{array}{l}\text { Exer- } \\
\text { cise }\end{array}$ & $\begin{array}{l}\text { Response } \\
\text { to } \\
\text { exercise }\end{array}$ & $\begin{array}{c}\text { Re- } \\
\text { covery }\end{array}$ & & & & \\
\hline $\begin{array}{l}\text { Mean pre-op. } \\
\text { Mean post-op. } \\
\text { Mean diff. } \\
\text { P }\end{array}$ & $\begin{array}{c}52 \pm 7 \\
68 \pm 11 \\
- \\
-\end{array}$ & $\begin{array}{c}34 \pm 4 \\
43 \pm 8 \\
- \\
-\end{array}$ & $\begin{array}{c}-19 \pm 7 \\
-24 \pm 8 \\
-7 \pm 8 \\
0.4\end{array}$ & $\begin{array}{c}52 \pm 7 \\
63 \pm 7 \\
- \\
-\end{array}$ & $\begin{array}{c}80 \pm 16 \\
126 \pm 20 \\
- \\
-\end{array}$ & $\begin{array}{c}60 \pm 10 \\
92 \pm 16 \\
-\end{array}$ & $\begin{array}{c}-32 \pm 16 \\
-34 \pm 10 \\
-0.2 \pm 13 \\
>0.9\end{array}$ & $\begin{array}{c}82 \pm 21 \\
125 \pm 18 \\
- \\
-\end{array}$ & & & & \\
\hline
\end{tabular}

* In occasional cases, measurements of some variables were available preoperatively but not postoperatively, or vice versa. These are included in the mean pre- and postoperative data. Occasional discrepancies between the means, the individual differences, and the difference between average pre- and postoperative values for the group are due to inclusion of these occasional unpaired data.

$\mathrm{Hg}$ (64 per cent) before, and $10 \mathrm{~mm}$. $\mathrm{Hg}$ ( 53 per cent) after operation, a change which was not statistically significant. However, in three patients the rise in "PC" pressure following exercise was considerably lower (12 to $22 \mathrm{~mm}$. $\mathrm{Hg}$ ) after operation.

Right ventricular end-diastolic pressure. The average right ventricular end-diastolic (R.V.D.) pressure at rest before operation was $10 \mathrm{~mm}$. $\mathrm{Hg}$ and after operation $7 \mathrm{~mm}$. Hg. After exercise the average R.V.D. pressure was $15 \mathrm{~mm}$. Hg preoperatively and $12 \mathrm{~mm}$. $\mathrm{Hg}$ postoperatively. In response to exercise the R.V.D. pressure increased on the average $8 \mathrm{~mm}$. $\mathrm{Hg}$ before operation and only $4 \mathrm{~mm}$. Hg after operation, a change which was just statistically significant.

\section{Arterial pressure, heart rate, stroke index}

The average mean arterial pressure at rest, 102 $\mathrm{mm}$. $\mathrm{Hg}$ preoperatively and $95 \mathrm{~mm}$. $\mathrm{Hg}$ postoperatively, showed no significant change. However, postoperatively two patients, A. L. and C. J. (the latter patient having associated arterial hy- pertension) showed appreciable falls in the mean arterial pressure of 20 and $50 \mathrm{~mm}$. $\mathrm{Hg}$, respectively. Likewise, the response of the arterial pressure to exercise was not significantly different before and after operation.

The heart rate at rest was 77 beats per min. preoperatively and 76 beats per min. postoperatively for the group, while after exercise it was 120 beats per min. before and 107 beats per min. after operation. Similarly, in response to exercise the heart rate was, on the average, 12 beats per min. lower after operation. These changes were not statistically significant.

At rest the slight rise in the stroke index after operation was not significant. However, with exercise the average stroke index was $31 \mathrm{ml}$. per min. per $\mathrm{M}^{2}$ preoperatively and $41 \mathrm{ml}$. per min. per $\mathrm{M}^{2}$ postoperatively. Likewise, the increase in stroke index in response to exercise was on the average $8 \mathrm{ml}$. per min. per $\mathrm{M}^{2}$ more after than before operation. These increases in stroke index were significant and reflected the associated increases in cardiac index and reductions in heart rate after operation. 


\section{Vascular resistances (in units of dynes seconds $\mathrm{cm}^{-5}$ )}

The "total pulmonary" resistance, which includes the calculated resistance of the mitral valve itself, is normally under 200 units at rest. Postoperatively there was a considerable and statistically significant decrease in this resistance as calculated from the data obtained during rest, the average declining from 940 to 530 units. The resistances thus remained elevated above normal even after operation. The resistances calculated from the measurements obtained following exercise likewise dropped (on the average from 1,210 to 630 units) after operation, which is also highly significant statistically. While some patients had only negligible changes in resistance as a result of operation, the direction of the change was uniformly downward.

In response to exercise, "total pulmonary" resistance in normal subjects usually decreases. In eight patients in whom data were obtained both at rest and after exercise pre- and postoperatively, the resistance rose considerably following exercise. The average rise was, however, much less after operation (90 units) than before (300 units). This change was highly significant statistically.

"Pulmonary arteriolar" resistance at rest is normally up to 100 units, and it usually decreases with exercise. In this group, the highly elevated preoperative resting value (average 470 units) declined after operation to 230 . Following exercise the "pulmonary arteriolar" resistance was increased (average 650 units) preoperatively, and declined to 280 units after operation. Although most of the patients had lower "pulmonary arteriolar" resistances both at rest and following exercise after operation, the average decreases for the entire group lacked statistical significance. However, the average increase in "pulmonary arteriolar" resistance in response to exercise was 170 units before operation and was only 40 units after operation, a reduction which was statistically significant.

The above calculated resistances indicate that about half the decrease in resistance opposing the right ventricle, both at rest and after exercise, can be accounted for in terms of a reduction in the resistance of the pulmonary vessels, and the other half in terms of a lessening of the resistance beyond the lungs, namely of the mitral valve itself.
The "total peripheral" resistance after operation tended to be less at rest and following exercise than before operation, but these changes were small and not statistically significant.

\section{Ventricular work against pressure (in units of kg. M. per min. per $M^{2}$ )}

The work of the right ventricle against pressure (normally less than 1.0 unit) was abnormally high at rest preoperatively (1.5 units) and declined postoperatively to 1.2 units. With exercise, the average right ventricular work rose to 3.2 units preoperatively and to only 2.5 units postoperatively. Thus, the considerable decrease in pulmonary arterial pressure with operation tends to reduce the amount of work by the right ventricle to a greater degree than the slight rise in cardiac output that acts to increase it. As a result of the balances of these two factors, the net effect is a reduction in the right ventricular work, but one which is not statistically significant.

The work of the left ventricle against pressure at rest tended to rise slightly after operation because the increase in cardiac output was greater than the fall in peripheral arterial pressure. However, neither the average values of the left ventricular work at rest nor the responses to exercise after operation were significantly greater than before operation.

\section{Renal responses before and after mitral valvulo- plasty}

Individual renal data are presented in Table IV, and group means and their statistical analysis in Table III.

At rest. Preoperatively all the patients had an abnormally low renal plasma flow (average 318 ml. per min. per $1.73 \mathrm{M}^{2}$ ) while five patients (C. S., E. M., C. M., E. C., A. C.) also had lower than normal glomerular filtration rates (mean value for the entire group was $100 \mathrm{ml}$. per min. per $1.73 \mathrm{M}^{2}$ ). Although after operation the renal plasma flow and glomerular filtration rate at rest showed significant increases to or towards normal in three patients, C. S., E. T., E. C., the postoperative renal plasma flow (mean $370 \mathrm{ml}$. per min. per $1.73 \mathrm{M}^{2}$ ) and glomerular filtration rate (mean $117 \mathrm{ml}$. per min. per $1.73 \mathrm{M}^{2}$ ) for the group as a whole did not show a statistically significant increase. Since intake of dietary salt was not regu- 
lated, the influence of the operation upon the excretion of electrolytes at rest could not be accurately evaluated.

During exercise. During the exercise period the mean renal plasma flow was $195 \mathrm{ml}$. per min. per $1.73 \mathrm{M}^{2}$ preoperatively and $283 \mathrm{ml}$. per min. per $1.73 \mathrm{M}^{2}$ postoperatively. Before operation the renal plasma flow decreased in response to exercise, on the average, $138 \mathrm{ml}$. per min. per 1.73 $\mathrm{M}^{2}$ or 43 per cent, and the glomerular filtration rate fell, on the average, $38 \mathrm{ml}$. per min. per 1.73 $\mathrm{M}^{2}$, or 38 per cent. After operation, exercise produced only about half as much decrease in the renal plasma flow (mean reduction $87 \mathrm{ml}$. per min. per $1.73 \mathrm{M}^{2}$, or 23 per cent), but this alteration just lacked statistical significance for the group. Likewise, the glomerular filtration rate was low- ered only half as much after operation (mean reduction $26 \mathrm{ml}$. per min. per $1.73 \mathrm{M}^{2}$, or 22 per cent), but again the change lacked statistical significance for the group.

Preoperatively, associated with the reduction caused by exercise in renal plasma flow and glomerular filtration rate, there were decreases in urine flow and the excretion of sodium, and usually of chloride, except in one patient, C. S. Likewise, after operation in all but one patient, J. C., there was a decrease in sodium excretion during the exercise period. In general, except for patient E. T., the reduction in sodium excretion during the exercise period paralleled the fall in the renal plasma flow and glomerular filtration rate. However, in response to exercise the decrease in sodium excretion was not significantly different be-

TABLE IV

Individual renal data

\begin{tabular}{|c|c|c|c|c|c|c|c|c|c|c|}
\hline $\begin{array}{c}\text { Patient } \\
\text { Age Sex } \\
\text { Body surface } \\
\text { area } \\
\left(M^{2}\right)\end{array}$ & $\begin{array}{c}\text { Time } \\
\text { relative to } \\
\text { operation }\end{array}$ & Procedure & Periods & $\begin{array}{c}\mathrm{C}_{\mathrm{PAH}} \\
\operatorname{ml./\operatorname {min}./} \\
1.73 \mathrm{M}^{2}\end{array}$ & $\begin{array}{c}\mathrm{C}_{\mathrm{IN}} \\
\operatorname{ml} . / \min _{1} / \\
1.73 \mathrm{M}^{2}\end{array}$ & F.F. & $\underset{m l . / m i n}{\mathrm{UV}}$ & $\underset{\substack{\mathrm{U}_{\mathrm{NaV}} \\
\text { min. }}}{\text { ma./ }}$ & $\underset{\substack{\mathrm{UclV} \\
\text { min. }}}{\text { mol/ }}$ & 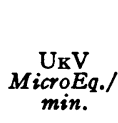 \\
\hline \multirow{3}{*}{$43{ }_{1.96}^{\mathrm{J} \cdot \mathrm{C} .} \mathrm{M}$} & \multirow{3}{*}{$\begin{array}{l}16 \text { wks. } \\
\text { Post-op. }\end{array}$} & \multirow{3}{*}{$\begin{array}{c}\text { Control } \\
\text { Exercise } \\
6 \text { min. } \\
\text { Recovery }\end{array}$} & $1-5$ & 451 & 124 & 28 & 11.0 & 132 & 126 & 52 \\
\hline & & & 6 & 336 & 100 & 30 & 5.5 & 142 & 138 & 39 \\
\hline & & & $7-9$ & 384 & 113 & 29 & 6.0 & 166 & 180 & 56 \\
\hline \multirow{2}{*}{$36{ }_{1.64}^{\text {A. L. }} F$} & \multirow[t]{3}{*}{ Pre-op. } & \multirow{6}{*}{$\begin{array}{l}\text { Control } \\
\text { Exercise } \\
7 \text { min. } \\
\text { Recovery } \\
\text { Control } \\
\text { Exercise } \\
9 \frac{1}{2} \text { min. } \\
\text { Recovery }\end{array}$} & $1-5$ & 330 & 114 & 35 & 10.0 & 121 & 87 & 35 \\
\hline & & & 6 & 229 & 97 & 42 & 2.7 & 67 & 75 & 35 \\
\hline \multirow{4}{*}{1.63} & & & $7-9$ & 306 & 115 & 38 & 10.0 & 282 & 170 & 64 \\
\hline & \multirow{3}{*}{$\begin{array}{l}9 \text { wks. } \\
\text { Post-op. }\end{array}$} & & $1-4$ & 305 & 121 & 40 & 12.0 & 131 & 128 & 45 \\
\hline & & & 5 & 189 & 84 & 44 & 5.2 & 61 & 78 & 32 \\
\hline & & & $6-7$ & 318 & 139 & 44 & 9.5 & 130 & 135 & 45 \\
\hline \multirow{6}{*}{$40{ }_{1.63}^{\text {C. J. }}{ }^{*} \mathrm{~F}$} & \multirow[t]{3}{*}{ Pre-op. } & \multirow{6}{*}{$\begin{array}{c}\text { Control } \\
\text { Exercise } \\
5 \text { min. } \\
\text { Recovery } \\
\text { Control } \\
\text { Exercise } \\
5 \text { min. } \\
\text { Recovery }\end{array}$} & $1-3$ & 347 & - & - & 2.5 & 96 & 95 & 75 \\
\hline & & & 4 & 124 & - & - & 0.15 & 33 & 40 & 24 \\
\hline & & & $5-6$ & 283 & - & - & 0.91 & 63 & 76 & 60 \\
\hline & \multirow{3}{*}{$\begin{array}{l}2 \text { wks. } \\
\text { Post-op. }\end{array}$} & & $1-3$ & 378 & - & - & 3.2 & 56 & 83 & 111 \\
\hline & & & 4 & 290 & - & - & 1.3 & 24 & 28 & 34 \\
\hline & & & $5-7$ & 394 & - & - & 1.3 & 63 & 74 & 70 \\
\hline \multirow{6}{*}{ 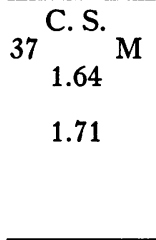 } & \multirow[t]{3}{*}{ Pre-op. } & \multirow{6}{*}{$\begin{array}{l}\text { Control } \\
\text { Exercise } \\
8 \mathrm{~min} . \\
\text { Recovery } \\
\text { Control } \\
\text { Exercise } \\
9 \frac{1}{2} \text { min. } \\
\text { Recovery }\end{array}$} & $1-4$ & 256 & 95 & 37 & 5.0 & 80 & 73 & 39 \\
\hline & & & 5 & 220 & 82 & 37 & 3.9 & 102 & 101 & 44 \\
\hline & & & $6-7$ & 267 & 104 & 39 & 0.94 & 74 & 59 & 41 \\
\hline & \multirow{3}{*}{$\begin{array}{l}28 \text { wks. } \\
\text { Post-op. }\end{array}$} & & $1-4$ & 381 & 131 & 34 & 11.0 & 116 & 98 & 88 \\
\hline & & & 5 & 318 & 106 & 33 & 2.8 & 89 & 97 & 63 \\
\hline & & & $6-7$ & 339 & 110 & 32 & 3.2 & 170 & 152 & 80 \\
\hline \multirow{6}{*}{$48_{1.66^{\text {E. M. }}}^{\text {F }}$} & \multirow[t]{3}{*}{ Pre-op. } & \multirow{6}{*}{$\begin{array}{c}\text { Control } \\
\text { Exercise } \\
2 \frac{1}{2} \mathrm{~min} . \\
\text { Recovery } \\
\text { Control } \\
\text { Exercise } \\
6 \text { min. } \\
\text { Recovery }\end{array}$} & $1-5$ & 267 & 98 & 37 & 7.0 & 127 & 79 & 35 \\
\hline & & & 6 & 94 & 42 & 44 & 2.7 & 54 & 36 & 17 \\
\hline & & & 7 & 181 & 83 & 46 & 0.40 & 29 & 33 & 27 \\
\hline & \multirow{3}{*}{$\begin{array}{l}20 \text { wks. } \\
\text { Post-op. }\end{array}$} & & $1-4$ & 215 & 101 & 47 & 7.3 & 132 & 119 & 37 \\
\hline & & & 5 & 146 & 55 & 37 & 1.4 & 84 & 84 & 22 \\
\hline & & & $6-7$ & 199 & 87 & 38 & 2.1 & 119 & 114 & 49 \\
\hline
\end{tabular}


TABLE IV-Continued

\begin{tabular}{|c|c|c|c|c|c|c|c|c|c|c|}
\hline $\begin{array}{c}\text { Patient } \\
\text { Age } \\
\text { Body surface } \\
\text { area } \\
\left(M^{2}\right)\end{array}$ & $\begin{array}{c}\text { Time } \\
\text { relative to } \\
\text { operation }\end{array}$ & Procedure & Periods & 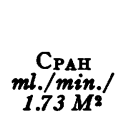 & 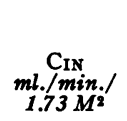 & F.F. & $\underset{m l . / m i n}{\mathrm{UV}}$ & 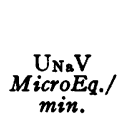 & $\underset{\substack{\text { MicrolE.I./ } \\
\text { min. }}}{\mathrm{UIV}}$ & $\underset{\substack{\mathrm{UKV} \\
\text { MicroEg./I } \\
\text { min. }}}{\cos }$ \\
\hline \multirow{3}{*}{$\begin{array}{c}\text { C. M. } \\
1.69 \\
1.69\end{array}$} & Pre-op. & \multirow{3}{*}{$\begin{array}{l}\text { Control } \\
\text { Exercise } \\
8 \text { min. } \\
\text { Recovery } \\
\text { Control } \\
\text { Exercise } \\
7 \text { min. } \\
\text { Recovery }\end{array}$} & $\begin{array}{c}1-5 \\
6\end{array}$ & $\begin{array}{l}381 \\
236\end{array}$ & $\begin{array}{r}106 \\
75\end{array}$ & $\begin{array}{l}28 \\
32\end{array}$ & $\begin{array}{l}5.8 \\
1.1\end{array}$ & $\begin{array}{r}170 \\
83\end{array}$ & $\begin{array}{r}150 \\
66\end{array}$ & $\begin{array}{l}79 \\
44\end{array}$ \\
\hline & \multirow[t]{2}{*}{$\begin{array}{l}32 \text { wks. } \\
\text { Post-op. }\end{array}$} & & $\begin{array}{l}7-8 \\
1-3 \\
4-5\end{array}$ & $\begin{array}{l}430 \\
303 \\
255\end{array}$ & $\begin{array}{r}124 \\
91 \\
84\end{array}$ & $\begin{array}{l}29 \\
30 \\
32\end{array}$ & $\begin{array}{l}3.9 \\
1.9 \\
2.4\end{array}$ & $\begin{array}{r}130 \\
135 \\
94\end{array}$ & $\begin{array}{l}114 \\
158 \\
123\end{array}$ & $\begin{array}{l}77 \\
77 \\
67\end{array}$ \\
\hline & & & 6 & 291 & 100 & 34 & 3.5 & 107 & 137 & 88 \\
\hline \multirow{3}{*}{${ }_{22}{ }_{1.49}^{\text {E. T. }}$ F } & Pre-op. & \multirow{3}{*}{$\begin{array}{l}\text { Control } \\
\text { Exercise } \\
6 \frac{1}{2} \text { min. } \\
\text { Recovery } \\
\text { Control } \\
\text { Exercise } \\
5 \text { min. } \\
\text { Recovery }\end{array}$} & $\begin{array}{c}1-3 \\
4\end{array}$ & $\begin{array}{l}486 \\
324\end{array}$ & $\begin{array}{r}127 \\
36\end{array}$ & $\begin{array}{l}26 \\
11\end{array}$ & $\begin{array}{l}4.5 \\
0.43\end{array}$ & $\begin{array}{l}47 \\
34\end{array}$ & $\begin{array}{l}64 \\
41\end{array}$ & $\begin{array}{l}51 \\
27\end{array}$ \\
\hline & $\begin{array}{l}17 \text { wks. } \\
\text { Post-op. }\end{array}$ & & $\begin{array}{c}5-6 \\
1-3 \\
4\end{array}$ & $\begin{array}{l}392 \\
689 \\
634\end{array}$ & $\begin{array}{l}115 \\
169 \\
165\end{array}$ & $\begin{array}{l}29 \\
25 \\
26\end{array}$ & $\begin{array}{l}0.41 \\
1.0 \\
0.48\end{array}$ & $\begin{array}{l}28 \\
85 \\
70\end{array}$ & $\begin{array}{l}42 \\
67 \\
54\end{array}$ & $\begin{array}{l}37 \\
16 \\
16\end{array}$ \\
\hline & & & $5-6$ & 625 & 173 & 28 & 0.34 & 81 & 64 & 22 \\
\hline \multirow{4}{*}{$\begin{array}{c}\text { E. C. } \\
\text { Fo } \\
1.43 \\
1.44\end{array}$} & Pre-op. & \multirow{4}{*}{$\begin{array}{l}\text { Control } \\
\text { Exercise } \\
\text { Recovery } \\
\text { Control } \\
\text { Exercise } \\
7 \text { min. } \\
\text { Recovery }\end{array}$} & $1-3$ & 206 & 82 & 40 & 0.28 & 6 & 12 & 35 \\
\hline & & & - & - & - & - & - & - & - & 二 \\
\hline & $\begin{array}{l}2 \text { wks. } \\
\text { Post-op. }\end{array}$ & & $\begin{array}{c}1-4 \\
5\end{array}$ & $\begin{array}{l}368 \\
269\end{array}$ & $\begin{array}{r}127 \\
94\end{array}$ & $\begin{array}{l}35 \\
35\end{array}$ & $\begin{array}{l}6.3 \\
4.9\end{array}$ & $\begin{array}{l}286 \\
192\end{array}$ & $\begin{array}{l}266 \\
181\end{array}$ & $\begin{array}{r}106 \\
84\end{array}$ \\
\hline & & & $6-8$ & 385 & 125 & 33 & 2.5 & 226 & 216 & 77 \\
\hline \multirow{3}{*}{$\begin{array}{r}40 \begin{array}{c}\text { A. C. } \\
2.10 \\
2.08\end{array} \\
\text { M }\end{array}$} & Pre-op. & \multirow{3}{*}{$\begin{array}{l}\text { Control } \\
\text { Exercise } \\
7 \text { min. } \\
\text { Recovery } \\
\text { Control } \\
\text { Exercise } \\
8 \text { min. } \\
\text { Recovery }\end{array}$} & $\begin{array}{c}1-3 \\
4\end{array}$ & $\begin{array}{l}268 \\
140\end{array}$ & $\begin{array}{l}76 \\
54\end{array}$ & $\begin{array}{l}28 \\
39\end{array}$ & $\begin{array}{l}3.4 \\
0.80\end{array}$ & $\begin{array}{l}780 \\
204\end{array}$ & $\begin{array}{l}778 \\
216\end{array}$ & $\begin{array}{l}66 \\
44\end{array}$ \\
\hline & $\begin{array}{l}6 \text { wks. } \\
\text { Post-op. }\end{array}$ & & $\begin{array}{c}5-7 \\
1-4 \\
5\end{array}$ & $\begin{array}{l}197 \\
242 \\
108\end{array}$ & $\begin{array}{l}69 \\
72 \\
36\end{array}$ & $\begin{array}{l}35 \\
29 \\
33\end{array}$ & $\begin{array}{l}1.0 \\
6.6 \\
2.5\end{array}$ & $\begin{array}{r}221 \\
51 \\
14\end{array}$ & $\begin{array}{r}242 \\
85 \\
41\end{array}$ & $\begin{array}{l}56 \\
79 \\
34\end{array}$ \\
\hline & & & $6-8$ & 225 & 84 & 37 & 4.4 & 27 & 55 & 76 \\
\hline \multirow{4}{*}{$39 \begin{array}{c}\text { A. S. } \\
1.46 \\
1.46\end{array}$} & Pre-op. & \multirow{4}{*}{$\begin{array}{l}\text { Control } \\
\text { Exercise } \\
\text { Recovery } \\
\text { Control } \\
\text { Exercise } \\
6 \text { min. } \\
\text { Recovery }\end{array}$} & $1-3$ & 589 & 121 & 20 & 4.7 & 148 & 120 & 35 \\
\hline & & & - & - & - & - & - & - & - & - \\
\hline & $\begin{array}{l}16 \text { wks. } \\
\text { Post-op. }\end{array}$ & & $\begin{array}{c}1-3 \\
4\end{array}$ & $\begin{array}{l}513 \\
265\end{array}$ & $\begin{array}{r}133 \\
78\end{array}$ & $\begin{array}{l}26 \\
29\end{array}$ & $\begin{array}{l}11.0 \\
0.08\end{array}$ & $\begin{array}{r}148 \\
21\end{array}$ & $\begin{array}{r}105 \\
17\end{array}$ & $\begin{array}{l}31 \\
11\end{array}$ \\
\hline & & & 5 & 355 & 110 & 31 & 0.20 & 7 & 8 & 15 \\
\hline
\end{tabular}

* Patient was given $300 \mathrm{ml} .5$ per cent $\mathrm{NaCl}$ I.V.

fore and after operation. The significant decrease in the excretion of potassium during exercise both before and after operation tended to parallel the changes in the glomerular filtration rate. The magnitude of the decrease in the excretion of potassium in response to exercise was not significantly different preoperatively and postoperatively.

Recovery from exercise. Persistently lower values of glomerular filtration rate and renal plasma flow lasting from 13 to 45 minutes after exercise were observed in four patients (A. C., C. J., E. M., and E. T.) before operation. Two of these patients (E. M. and E. T.) developed clinical pulmonary edema during exercise. Three other patients (A. L., C. S., C. M.), none of whom developed pulmonary edema, had a prompt restor- ation in the renal hemodynamic measurements after exercise and a return to control values within 22 to 45 minutes. Postoperatively, the renal plasma flow tended to return more rapidly and completely to the pre-exercise values during the same period of recovery.

Before operation all except two patients (A. L., C. S.) had reductions in sodium, chloride and usually water excretion during exercise which persisted during the recovery period. In the recovery phase sodium and water retention in most of the patients appeared to be independent of the changes in the glomerular filtration rate, since the latter measurements frequently had already returned to the control values. After operation all patients recovered more readily from the exercise- 
induced reductions of sodium excretion except for three patients (E. C., C. M., A. C.) who had a persistent decrease in sodium excretion after exercise.

\section{DISCUSSION}

There was no consistent relationship between the cardiohemodynamic and the renal functional abnormalities observed in these patients before or after operation. No correlation could be found to exist between the absolute levels of the cardiac output and the renal plasma flow and glomerular filtration rate at rest or during exercise. After mitral valvuloplasty three patients (C. S., E. T., E. C.) showed significant increases in renal plasma flow at rest, and four (C. J., E. M., C. M., E. T.) had smaller decreases in renal plasma flow during exercise. Of the two patients (C. S., E. T.) who had appreciable increases in the glomerular filtration rate at rest, one (E. T.) had a smaller decrease in the glomerular filtration rate in response to exercise after operation. However, these changes in renal plasma flow and glomerular filtration rate were not invariably related to improvement in cardiac output. For example, one patient in the group (C. S.) who had the greatest increase in cardiac output in response to exercise postoperatively had a fall in the renal hemodynamic measurements equal to that found preoperatively. On the other hand, the cardiac output response to exercise was similar before and after operation in patient $\mathrm{C}$. J. whereas the renal plasma flow during the exercise period showed a lesser fall after than before operation.

Prior to operation two patients (E. M., E. T.) developed cardiac asthma during, exercise with markedly elevated "pulmonary capillary" pressures, and also had marked reductions in glomerular filtration rate and renal plasma flow and persistent decreases in the excretion of salt and water. After operation they had no cardiac asthma with exercise and smaller decreases in renal plasma flow, glomerular filtration rate, or excretion of salt and water. However, the decreases in renal plasma flow and glomerular filtration rate during the exercise period in the group as a whole could not be correlated with any abnormal increases in mixed arteriovenous oxygen difference or levels of central vascular pressures before or after operation.
Preoperatively the return of the renal hemodynamic measurements to or towards control levels after the exercise period was not consistently related to the prior increase in cardiac output during exercise. However, in some patients after operation there appeared to be an association between improvement in the cardiac output responses to exercise and speed of return of the renal hemodynamic measurements to control levels after exercise. In several patients before operation the decreases in the excretion of sodium and water persisted for some time after exercise. In some cases they continued after the glomerular filtration rates had returned to control values. These decreases were therefore attributed, at least in part, to increased renal tubular reabsorption of salt and water. After operation, however, these decreases in renal function in general persisted for shorter periods. In only one patient (A. C.) in whom an increased renal tubular reabsorption of sodium persisted after exercise did a marked increase in the hematocrit (suggesting a decrease in blood volume with hemoconcentration) occur during exercise both before and after mitral valvuloplasty. It is possible that these two factors may have directly influenced the renal tubular reabsorption of sodium. In other patients during exercise, smaller increases in hematocrit occurred and appeared to contribute little, if at all, to the retention of sodium.

None of the patients after operation had a return to normal of their cardiohemodynamic or their renal functions in response to exercise. Several patients, on the other hand, had a return of individual functions to or almost to normal values at rest. In some, the reductions in urine flow, sodium excretion, renal plasma flow, and glomerular filtration rate were less marked especially in response to exercise than before operation. Likewise in some, the cardiohemodynamic abnormalities were less marked both at rest and in response to exercise than before operation. However, there was no consistent parallelism between these two types of improvement, particularly if measurements of only a single renal or circulatory function was considered. However, there was a fairly consistent trend for those patients with the more normal cardiohemodynamic responses to exercise to show the more normal renal responses.

In general, symptomatic improvement paralleled 
improvement in the cardiohemodynamic, or the renal function, or both. However, in some patients marked subjective improvement was noted with little change in either the cardiovascular or renal measurements. In fact, one patient (A. S.) in whom the left auricle was opened but no fracture of the mitral valve accomplished because of technical difficulties had marked symptomatic improvement for about six months without any significant alterations in cardiovascular or renal function.

Some reports on physiologic data obtained from patients with mitral stenosis following mitral valvuloplasty indicate that maximum improvement in the hemodynamic measurements may not appear immediately (24), or early, but usually several months after operation (25-27). The hemodynamic observations in our patients, who were studied early after operation (2 to 32 weeks), were believed at the time to represent maximum states of improvement. Cardiac catheterizations have been repeated in a majority of them one and one-half to two years postoperatively, and the hemodynamic responses in some cases found to be more normal and in others more abnormal than the initial postoperative studies reported here, and a few, actually more abnormal than the preoperative values $(28)$.

\section{SUMMARY}

Cardiovascular and renal measurements were made at rest and following exercise in nine patients with mitral stenosis before and within 2 to 32 weeks after mitral valvuloplasty. Cardiohemodynamic measurements after operation showed a slight, but not statistically significant, increase in the cardiac index, and a significant increase in the stroke index, since the heart rates tended to be slower. The pulmonary arterial and "pulmonary capillary" pressures were significantly lower. The right ventricular end-diastolic pressure was not significantly reduced at rest, but after exercise it was significantly less than before operation. The calculated "total pulmonary" resistance (which includes the resistance imposed by the mitral valve) was significantly reduced, but the "pulmonary arteriolar" resistance was not. The increase in the "pulmonary arteriolar" resistance in response to exercise was not so great after operation. The resistance opposing the right ventricle was decreased after operation apparently because of approximately equal reductions in the resistance of the pulmonary vessels and that imposed by the stenosis of the mitral valve. There was a slight reduction in the work of the right ventricle and a slight increase in the work of the left ventricle after operation but neither was statistically significant.

Preoperative resting renal plasma flow was approximately half and glomerular filtration rate about four-fifths of normal values. After operation the resting renal plasma flow and glomerular filtration rate were both increased, on the average, by about one-sixth, the latter approaching normal. In response to exercise the renal plasma flow fell, on the average, 43 per cent before operation and only one-half that much after operation, a significant improvement. The glomerular filtration rate decreased in response to exercise, on the average, 38 per cent before operation and only about twothirds that much after operation. This improvement was not statistically significant. The considerable decreases in urine flow, excretion of sodium, chloride and potassium in response to exercise, which tended to parallel the changes in the glomerular filtration rate, were not significantly different in magnitude after operation as compared with before. Although all the renal functions tended to recover from exercise more rapidly after operation, the mean data showed no significant differences from the preoperative responses.

In this group of patients mitral valvuloplasty produced varying degrees of improvement in the cardiovascular and renal functions both at rest and after exercise. In none of the patients, however, did the cardio-renal hemodynamic measurements return to normal values. A general improvement in cardiovascular function in individual patients was sometimes associated with increases in renal plasma flow, glomerular filtration rate and the ability to excrete salt and water during and after exercise. However, postoperative increases in salt and water excretion could not be consistently correlated with any specific change in cardiovascular or renal function, or both.

\section{ACKNOWLEDGMENT}

The authors wish to thank Dr. Robert W. Wilkins for his excellent suggestions and criticisms. 


\section{REFERENCES}

1. Dexter, L., Gorlin, R., Lewis, B. M., Haynes, F. W., and Harken, D. E., Physiologic evaluation of patients with mitral stenosis before and after mitral valvuloplasty. Tr. Am. Climat. \& Clin. A., 1950, 62, 170.

2. Draper, A., Heimbecker, R., Daley, R., Carrol, D., Mudd, G., Wells, R., Falholt, W., Andrus, E. C., and Bing, R. J., Physiologic studies in mitral valvular disease. Circulation, 1951, 3, 531.

3. Lukas, D. S., and Dotter, C. T., Modifications of the pulmonary circulation in mitral stenosis. Am. J. Med., 1952, 12, 639.

4. Jordan, P., and Hellems, H. K., Mitral valve surgery. A critical analysis. Surg., Gynec. \& Obst., 1952, 95, 689.

5. Janton, O. H., Glover, R. P., O'Neill, T. J. E., Gregory, J. E., and Froio, G. F., Results of the surgical treatment for mitral stenosis. Analysis of one hundred consecutive cases. Circulation, 1952, 6, 321.

6. Hurwitt, E. S., Bloomberg, A., Aaron, R., Jezer, A., and Young, D., An appraisal of the surgical treatment of mitral stenosis. Ann. Surg., 1953, 138, 219.

7. Cosby, R. S., Griffith, G. C., Levinson, D. C., Zinn, W. J., Oblath, R. W., Dimitroff, S. P., and Herman, L. M., The physiologic evaluation of patients before and after mitral commissurotomy. Dis. of Chest, 1953, 23, 499.

8. Baranofsky, I. D., Haddy, F. J., Ferrin, A. L., and Borden, C. W., Cardiopulmonary dynamics of mitral stenosis. A clinical and experimental study. Surgery, 1953, 34, 347.

9. Curti, P. C., Cohen, G., Castleman, B., Scannell, J. G., Friedlich, A. L., and Meyers, G. S., Respiratory and circulatory studies in patients with mitral stenosis. Circulation, 1953, 8, 893.

10. Werkö, L., Biörck, G., Crafoord, C., Wulff, H., Krook, H., and Eliasch, H., Pulmonary circulatory dynamics in mitral stenosis before and after commissurotomy. Am. Heart J., 1953, 45, 477.

11. Merrill, A. J., Edema and decreased renal blood flow in patients with chronic congestive heart failure: evidence of "forward failure" as the primary cause of edema. J. Clin. Invest., 1946, 25, 389.

12. Eichna, L. W., Farber, S. J., Berger, A. R., Earle, D. P., Rader, B., Pellegrino, E., Albert, R. E., Alexander, J. D., Taube, H., and Youngwirth, S., Cardiovascular dynamics, blood volumes, renal functions and electrolyte excretions in the same patients during congestive heart failure and after recovery of cardiac decompensation. Circulation, $1953,7,674$.

13. Merrill, A. J., and Cargill, W. H., The effect of exercise on the renal plasma flow and filtration rate of normal and cardiac subjects. J. Clin. Invest., 1948, 27, 272.

14. Werkö, L., Varnauskas, E., Eliasch, H., Ek, J., Bucht, H., Thomasson, B., and Bergström, J.,
Studies on the renal circulation and renal function in mitral valvuluar disease. I. Effect of exercise. Circulation, 1954, 9, 687.

15. Judson, W. E., Hollander, W., Friedman, I. H., Hatcher, J. D., and Halperin, M. H., Studies on congestive failure: The effects of exercise, salt loading and of intravenous Apresoline on cardiorenal hemodynamics and the excretion of sodium and water. J. Clin. Invest., 1953, 32, 579.

16. Judson, W. E., Hatcher, J. D., Hollander, W., Halperin, M. H., and Friedman, I. H., Cardiorenal hemodynamics and excretion of sodium and water, at rest and during exercise, in patients with mitral stenosis before and after mitral valvuloplasty. Clin. Research Proc., 1953, 1, 75.

17. Gorlin, R., Haynes, F. W., Goodale, W. T., Sawyer, C. G., Dow, J. W., and Dexter, L., Studies of the circulatory dynamics in mitral stenosis. II. Altered dynamics at rest. Am. Heart J., 1951, 41, 30.

18. Haldane, J. S., Methods of Air Analysis. London, Griffin, 1912.

19. Van Slyke, D. D., and Neill, J. M., The determination of gases in blood and other solutions by vacuum extraction and manometric measurement. I. J. Biol. Chem., 1924, 61, 523.

20. Goldring, W., and Chasis, H., Hypertension and $\mathrm{Hy}-$ pertensive Disease. New York, The Commonwealth Fund, 1944.

21. Schreiner, G. E., Determination of inulin by means of resorcinol. Proc. Soc. Exper. Biol. \& Med., 1950, 74, 117.

22. Roe, J. H., Epstein, J. H., and Goldstein, N. P., A photometric method for the determination of inulin in plasma and urine. J. Biol. Chem., 1949, 178, 839.

23. Wilson, D. W., and Ball, E. G., A study of the estimation of chloride in blood and serum. J. Biol. Chem., 1928, 79, 221.

24. Lev, R., Connolly, D. C., Kirklin, J. W., and Wood, E. H., The immediate hemodynamic effects of mitral commissurotomy during surgery. Surg. Forum, 1953, 4, 13.

25. Baker, C., Brock, R. C., Campbell, M., and Wood, P., Valvotomy for mitral stenosis. A further report, on 100 cases. Brit. M. J., 1952, 1, 1043.

26. Ellis, F. H., Kirklin, J. W., Parker, R. L., Burchell, H. B., and Wood, E. H., Mitral commissurotomy. An over-all appraisal of clinical and hemodynamic results. Arch. Int. Med., 1954, 94, 774.

27. Sancetta, S. M., Mueller, R. P., and Gillespie, D. G., Preoperative, six-week, and twelve-month postoperative hemodynamic studies in patients undergoing mitral commissurotomy. J. Lab. \& Clin. Med., 1953, 42, 945.

28. Judson, W. E., Physiological evaluation of postoperative results of surgical treatment of mitral stenosis. Presented March 22, 1954 at a Symposium on Current Problems in Mitral Valvular Disease at the Massachusetts Memorial Hospitals, Boston, Mass., To be published. 